\title{
CLEC9A: linking necrosis and immunity
}

Necrotic cells can sometimes provoke an immune response in the absence of infection. In a study published in Nature, David Sancho and colleagues show that the recently described C-type lectin receptor CLEC9A (C-type lectin domain family 9A; also known as DNGR1) expressed by CD8 $\alpha^{+}$dendritic cells (DCs) couples the recognition of necrotic cells to the subsequent crosspresentation of dead-cell-associated antigens to $\mathrm{T}$ cells.

First, the authors investigated the ligands for CLEC9A by establishing a reporter system composed of the extracellular domains of CLEC9A

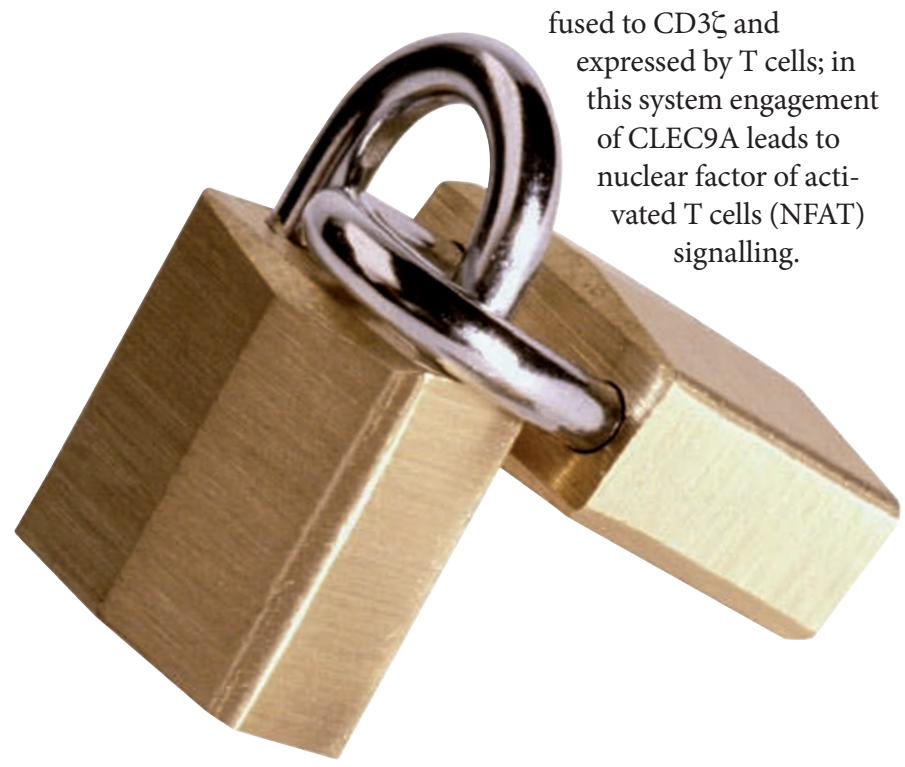

They initially observed basal NFAT signalling in cell cultures, which correlated with the number of dead cells in the culture. Irradiated mouse embryonic fibroblasts (MEFs) undergoing apoptotic cell death did not stimulate NFAT signalling until the cells underwent secondary necrosis at a later time point, indicating that CLEC9A recognizes a ligand (or ligands) exposed on necrotic and not apoptotic cells.

Next the authors generated a CLEC9A-deficient mouse. The development and phenotype of DCs from these mice were comparable to those of wild-type mice. Flow cytometric analysis of CLEC9A-deficient CD8 $\alpha^{+}$ DCs showed that CLEC9A was not required for binding to, or uptake of, necrotic cell material. However, when the authors induced necrosis in MEFs that expressed a non-secreted form of ovalbumin and added them to cultures of CLEC9A-deficient CD $8 \alpha^{+}$DCs plus ovalbumin-specific transgenic $\mathrm{T}$ cells, they found that the CLEC9A-deficient CD8 $\alpha^{+}$DCs were unable to cross-present deadcell-associated antigens to the T cells. Similarly, in in vivo assays, CLEC9A was required for efficient crosspresentation of antigens from dead cells. The cross-presentation function of CLEC9A was found to be dependent on signalling by the spleen tyrosine kinase (SYK) pathway. Because antibody-labelled CLEC9A does not localize to the lysosome, the authors propose that CLEC9A diverts cargo away from lysosomal compartments such that it is available for crosspresentation. So CLEC9A, a receptor expressed by DCs that recognizes a preformed ligand sequestered in healthy cells and exposed on necrotic but not apoptotic cells, is a classical 'danger' receptor.

This study shows that CLEC9A is important for conveying information from necrotic cells to T cells and defines a pathway by which adaptive immune responses can be initiated in the absence of infection.

Elaine Bell

ORIGINAL RESEARCH PAPER Sancho, D. et al. Identification of a dendritic cell receptor that couples sensing of necrosis to immunity. Nature 15 Feb 2009 (doi:10.1038/nature07750) FURTHER READING Matzinger, P. The danger model: a renewed sense of self. Science $\mathbf{2 9 6}$, 301-305 (2002) 